\title{
Effect of diffuse light scattering designs on the efficiency of dye solar cells: an integral optical and electrical description
}

\author{
Francisco Enrique Gálvez, ${ }^{1,3}$ Erno Kemppainen, ${ }^{2}$ Hernán Míguez, ${ }^{1, *}$ Janne Halme $^{2, *}$
}

1 Multifunctional Optical Materials Group, Instituto de Ciencia de Materiales de Sevilla, Consejo

Superior de Investigaciones Científicas-Universidad de Sevilla(US-CSIC), Américo Vespucio 49, 41092 Sevilla, Spain. E-mail: hernan@icmse.csic.es

2 Department of Applied Physics, Aalto University School of Science, P.O.Box 15100, FI-00076, AALTO, Finland. E-mail: janne.halme@aalto.fi

3 Agencia Estatal de Meteorología, Sede de Andalucía Occidental, Américo Vespucio s/n, 41092 Sevilla, Spain.

\begin{abstract}
Herein we present an integral optical and electrical theoretical analysis of the effect of different diffuse light scattering designs on the performance of dye solar cells. Light harvesting efficiencies and electron generation functions extracted from optical numerical calculations based on a Monte Carlo approach are introduced in a standard electron diffusion model to obtain the steady-state characteristics of the different configurations considered. We demonstrate that there is a strong dependence of the incident photon to current conversion efficiency, and thus of the overall conversion efficiency, on the interplay between the value of the electron diffusion length considered and the type of light scattering design employed, which determines the spatial dependence of the electron generation function. Other effects, like the influence of increased photoelectron generation on the photovoltage, are also discussed. Optimized scattering designs for different combinations of electrode thickness and electron diffusion length are proposed.
\end{abstract}

\section{Introduction}

Correct optical design is essential to achieve high light-to-electric energy conversion efficiency with solar cells, and the electrochemical dye solar cells (DSCs) based on dye-sensitized nanocrystalline $\mathrm{TiO}_{2}$ photoelectrode films ${ }^{1}$ are no exception. ${ }^{2}$ One of the most common principles is to try to boost the light absorption by the photovoltaic material in the spectral regions where it is 
weakest. In the case of DSCs, the most commonly used N719 dye does not absorb red or longer wavelengths well, which gives the dye its distinct reddish colour. To compensate for this, it has become a standard practice to use diffuse light scattering to increase the optical path length of the cell at red wavelengths. ${ }^{3,4,5,6,7}$ In fact, virtually all DSCs that exhibit above $10 \%$ efficiency incorporate such additional porous back-scattering film, ${ }^{8,9,10}$ to reflect any non-absorbed light back into the film. In another approach, light scattering particles ${ }^{11,12,13}$ or cavities ${ }^{14,15}$ are embedded inside the $\mathrm{TiO}_{2}$ photoelectrode film, to give rise to multiple scattering that increases optical path and thus probability of absorption. In both cases, the light harvesting efficiency, $\eta_{\mathrm{LH}}(\lambda)$, of the cell and thus its short circuit current density and energy conversion efficiency are enhanced. However, it is generally accepted that the former approach provides larger reinforcements of the optical absorption than the latter, an assumption that has so far been based on empirical evidence.

Besides increasing light harvesting, light scattering also modifies the light absorption profile in the photoelectrode film, departing it from the Beer-Lambert law. Although significant advances have been made in recent years towards a more complete theoretical description of dye solar cells, 161718192021 and in spite of the fact that the most efficient cells present strong diffuse scattering, most calculations performed today to evaluate or predict the steady-state characteristics of DSCs still include the classical electron generation profile function,

$$
g(x, \lambda)=\alpha_{A} \cdot \exp \left(-\alpha_{A} \cdot x\right)
$$

, which is valid only for non-scattering films. In this expression, $\alpha_{A}$ is the wavelength dependent extinction coefficient of the photoelectrode film. The effect of that approximation is minor as long as the electron diffusion length $(L)$, defined as the average distance travelled by the electrons before recombination, is larger than the electrode thickness $(d)$. In fact, under these circumstances, assuming an arbitrary form for $g(x, \lambda)$ to simulate any high performance dye sensitized solar cells should not have an effect on the final output since when $L \gg>d$ the total harvested photon flux is independent of the spatial distribution of light absorption. This seems to be the only case that has so far been considered when simulations are performed, so applying equation (1) would not yield an incorrect result. However, for cells in which $L \leq d$, only electrons that are generated within distance $L$ from the contact can be effectively collected, while most of those generated further in the film are lost by recombination. ${ }^{22}$ In these cases, the actual shape and value of $g(x, \lambda)$ must be considered, since it will largely determine the electron collection efficiency, $\eta_{\text {col }}$, and thus the overall performance. The beneficial presence of diffuse scattering was first theoretically analyzed by Usami 
${ }^{23}$ and by Ferber and Luther. ${ }^{24}$ Its effect on the electrical parameters of the cell were later addressed in a couple of sound theoretical papers, ${ }^{25,26}$ in which the actual $\eta_{\mathrm{LH}}(\lambda)$ and $g(x, \lambda)$ for different light scattering designs were calculated. However, as far as we know, the crucial interplay between electron diffusion length and the actual electron generation function has not been accounted for in calculations of overall DSC performance up to date. Similar type of light scattering simulations combined with an electron diffusion model have been reported to obtain quantum efficiencies in the case of 4,7 , and $10 \mu \mathrm{m}$ thick light scattering photoelectrode films and $7 \mu \mathrm{m}$ electron diffusion length. In that work, it was also concluded that relatively more light is absorbed next to the collecting contact in a light scattering film, compared to non-scattering film, which can be beneficial for electron collection. Nevertheless, the relative contributions to the predicted performance enhancements from the improved light absorption on one hand and from the increased electron collection on the other hand were not investigated. In the present contribution, we complement the work of Usami with this respect, and go beyond, by comparing the results to the case where a nonscattering absorber film is combined with a diffuse back scattering layer. Moreover, the results are discussed in terms of the overall energy conversion efficiency, in addition to quantum efficiency.

In this paper we use combined optical and electrical modeling to investigate how different commonly used light scattering designs influence the conversion of light into electric energy. Effects on both light harvesting and electron collection are taken into account. We demonstrate that diffuse scattering layers acting as back reflectors provide the largest achievable light harvesting efficiencies, which, for electron diffusion lengths longer than the electrode thickness, determines an optimum overall performance. However, when the electron diffusion length is shorter than the electrode thickness, embedding diffuse scattering particles in the nanocrystalline paste may yield a better output even when the light harvesting is not optimal, since $g(x, \lambda)$ largely increases at distances close to the substrate contact with respect to any other configuration, which boost the collection efficiency compensating for the smaller absorptance. Our results serve both to explain previous observations as well as to identify guidelines to optimize the use of light in different sorts of dye solar cells.

\section{Theoretical model and computational tools}

Schemes of the different DSC designs herein analyzed are drawn in Figure 1, namely, a standard cell made of a film of nanocrystalline titania sensitized with a standard N719 dye (Figure 1(a)); a cell in which diffuse scattering particles have been mixed with the nanocrystalline paste (Figure 1(b)); and, finally, a cell made of a layer of nanocrystalline titania onto which a layer of diffuse scattering 
particles has been deposited (Figure 1(c)). For the sake of conciseness we will refer to these three configurations as type I, II and III, respectively.

\section{Optical modeling}

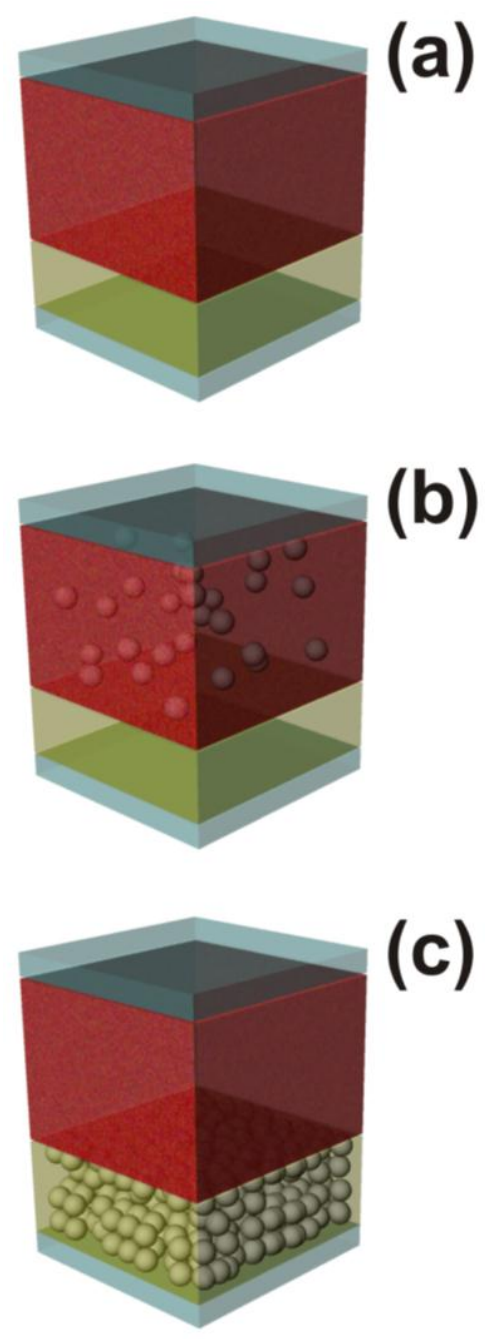

Figure 1. Drawings representing the different dye solar cell designs whose optical response is herein simulated. (a) Standard semitransparent cell (type I); (b) cell with an electrode embedding diffuse scattering particles (type II); and (c) cell made of a semitransparent electrode coupled to a diffuse scattering layer (type III).

To start, spectral light harvesting efficiency, $\eta_{\mathrm{LH}}(\lambda)$, and optical absorption profile functions, $g(x, \lambda)$, were calculated for the three types of DSCs. We have employed a Monte Carlo approach to simulate the trajectory of $10^{8}$ photons that enter the different cells under consideration. In this approximation, we are assuming that particles, be them included in the electrode or coupled to it as a back reflector, give rise to isotropic scattering, i.e., after undergoing a scattering event, photons can 
escape in all directions with equal probability. Thus we are neglecting Mie effects arising from the diffraction of wavelengths similar to the size of the particles, which could yield anisotropic angular distributions. This approximation is supported by the fact that the scattering particles typically used, be them mixed in the paste or as part of back diffusive mirrors, present a quite broad size distribution and their morphology is not homogeneous. These features yield a broad range of possible outcome directions of the individual scattering events that, statistically, can be represented as a randomized angular response.

Within this approximation, the analysis of the interaction between light and matter is reduced to the analysis of the effect of two parameters, namely $\ell_{\mathrm{A}}$ and $\boldsymbol{l}_{\mathrm{SC}}$, which are the absorption and scattering mean free paths, respectively, that is, the average distance photons travel before being absorbed in the standard transparent (non-diffusive) electrode and the average distance photons travel between scattering events when scattering particles are introduced in the problem. This approach resembles that used by Usami to simulate the effect of light confinement in DSC. ${ }^{26}$ Please notice that while $\ell_{\mathrm{A}}$ is wavelength dependent, $\ell_{\mathrm{SC}}$ is taken to be constant over the whole spectral range considered for both type I and type II cells to facilitate the comparison between them. Depending on the type of cell, two different cases are distinguished.

When scattering particles have been mixed in the dyed nanocrystalline paste (Type II, Figure 1(b)), once a photon enters the cell, the distance travelled before experiencing either absorption or scattering, $\ell$, is given by the expression

$$
\ell=-\ln [r] /\left(\alpha_{A}+\alpha_{S C}\right)
$$

where $r$ is a random number comprised in the range $0<\mathrm{r} \leq 1, \alpha_{A}=1 / \ell_{A}$ and $\alpha_{S C}=1 / \ell_{S C}$. The overall absorption coefficient $\alpha_{A}=\alpha_{d y e}+\alpha_{e l}$, where $\alpha_{d y e}$ and $\alpha_{d y e}$ are the wavelength dependent extinction coefficient of the dyed $\mathrm{TiO}_{2}$ photoelectrode film and the electrolyte in the pores of the film (with porosity taken into account), respectively. Although the only optical losses contributing to photocurrent are those resulting from light harvesting occurring at dye molecules, we have also estimated the unproductive absorption caused by the presence of the electrolyte, in order to account for this competing phenomenon. If $\ell$ is longer than the electrode thickness, $d$, then the photon is ballistically transmitted. If $\ell<d$ then a new random number, $r^{\prime}$, is generated. If $r^{\prime} \leq \alpha_{S C} /\left(\alpha_{A}+\alpha_{S C}\right)$, the photon is scattered; for $\alpha_{S C} /\left(\alpha_{A}+\alpha_{S C}\right)<r^{\prime} \leq\left(\alpha_{S C}+\alpha_{d y e}\right) /\left(\alpha_{A}+\alpha_{S C}\right)$, then it is absorbed by the dye, thus generating charges susceptible of contributing to the photocurrent; for $\left(\alpha_{S C}+\alpha_{d y e}\right) /\left(\alpha_{A}+\alpha_{S C}\right) \leq$ 
$r^{\prime}$, the photon is captured by the electrolyte and then lost. Being the dependence of $\boldsymbol{l}_{A}$ on wavelength taken from previously reported data, an increase of the value of $\ell_{\mathrm{SC}}$ would, in practical terms, represent a decrease of the concentration of scattering centers, assuming the type of particles employed is always the same. We have simulated the sensitivity of the response of the electrodes to the value of $\ell_{\mathrm{SC}}$ comprised in the range $1 \mu \mathrm{m}<\ell_{\mathrm{SC}}<11 \mu \mathrm{m}$ and found that the optimum case is that for which $\ell_{\mathrm{SC}}=3 \mu \mathrm{m}$. We will focus our later discussion on this particular example.
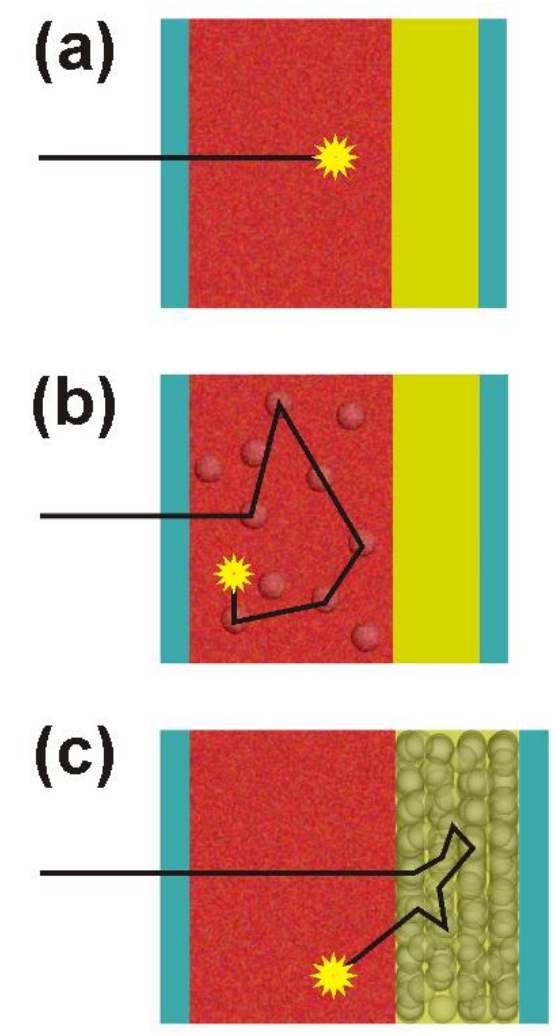

Figure 2. Selected simulated trajectories of a photon absorbed by (a) type I, (b) type II and (c) type III cells. Blue layers represent transparent conducting substrates, red ones dyed nanocrystalline electrodes and yellow ones the electrolyte overlayer typically present in DSCs (in this model, included as part of the scattering layer in type III cell).

For the case of a standard electrode coupled to a diffuse scattering layer (Type III, Figure 1(c)), no absorption is assumed to take place in the latter. Although, depending on the type of paste employed to build the scattering layer, there might be a certain contribution to the photocurrent from the absorption of the dye embedded in $\mathrm{it}^{27}$ we restrict ourselves to the case in which no absorption occurs in order to evaluate which one, among the different cells herein studied, present the optimized light scattering design. It must be taken into account that the scattering strength of such layer 
strongly affects the angular distribution of the light reflected back into the electrode. The cases herein selected cover a wide range of angular distributions for the light diffusely reflected back $(0.5$ $\left.\mu \mathrm{m}<l_{\mathrm{SC}}<4 \mu \mathrm{m}\right)$. The coupled diffuse scattering layer is taken to be $4 \mu \mathrm{m}$ thick, which is a standard value used in the field. In this case, the optimum $\ell_{\mathrm{SC}}$ is the shorter $(0.5 \mu \mathrm{m})$ since it gives rise to both higher back reflection and long path lengths for photons sent back into the absorbing electrode, which yield in turn higher probability of absorption. Again, this will be the case selected for further comparative analysis of the different cell efficiencies.

In Figure 2, selected simulated trajectories of a photon absorbed by (a) type I, (b) type II and (c) type III cells are drawn. They illustrate the different series of scattering events that yield longer optical paths and thus enhanced probability of absorption in each type of modified cell under consideration.

Using this model, we obtain the light harvesting efficiency $\eta_{\mathrm{LH}}(\lambda)$ and the electron generation profile $g(x, \lambda)$ for all three types of cell studied. We focused our study on the spectral region in which the absorption of the dye is noticeable, that is, 400-800 $\mathrm{nm}$. The spectral and spatial resolution considered is $\Delta \lambda=10 \mathrm{~nm}$ and $\Delta h=100 \mathrm{~nm}$, respectively. The spectral dependence of the dye and the electrolyte extinction coefficients is extracted from reported absorptance data of standard transparent cells. ${ }^{28}$ In all cases, the thickness of the absorbing electrode is taken to be $d=7.0$ microns. We neglected the specular reflectance arising from the interface between the transparent conducting oxide coating and the supporting glass plate to simplify the calculation. These are expected to contribute with a spectrally constant background of low intensity. The curves $\eta_{\mathrm{LH}}(\lambda)$ and $g(x, \lambda)$ serve in turn as input for the electrical modeling to attain the photocurrent, photovoltage and conversion efficiency of the cells as described next.

\section{Electrical modeling}

The short circuit current density of DSC photoelectrode $\left(i_{\mathrm{SC}}\right)$ can be expressed as

$$
i_{\mathrm{SC}}=q_{\mathrm{e}} \int_{\lambda_{\min }}^{\lambda_{\max }} \Phi(\lambda) \eta_{\mathrm{IPCE}}(\lambda) d \lambda
$$

where $\Phi$ is the incident photon flux $\left(\mathrm{m}^{-2} \mathrm{~s}^{-1}\right), q_{\mathrm{e}}$ is the electron charge, and the $\eta_{\mathrm{IPCE}}$ is the incidentphoton-to-collected-electron efficiency, which consists of the four partial efficiencies: light 
harvesting efficiency $\left(\eta_{\mathrm{LH}}\right)$, electron injection efficiency $\left(\eta_{\text {inj }}\right)$, dye regeneration efficiency $\left(\eta_{\text {reg }}\right)$, and electron collection efficiency $\left(\eta_{\text {col }}\right)$

$$
\eta_{\mathrm{IPCE}}(\lambda)=\eta_{\mathrm{LH}}(\lambda) \eta_{\mathrm{inj}}(\lambda) \eta_{\mathrm{reg}}(\lambda) \eta_{\mathrm{col}}(\lambda)
$$

Light scattering in the photoelectrode film affects primarily $\eta_{\mathrm{LH}}$ while it can be assumed to have no effect on $\eta_{\text {inj }}$ and $\eta_{\text {reg. }}$. As pointed out in the Introduction, light scattering can also have a secondary effect on $\eta_{\text {col }}$ when the electron collection form the film is less than perfect. This arises from the sensitivity of $\eta_{\mathrm{col}}$ on the electron generation profile $g(x)$ in the film, as predicted by the standard electron diffusion model. ${ }^{29}$

The continuity equation of conduction band electrons in the photoelectrode film can be written

$$
\frac{\partial n(x)}{\partial t}=\frac{\partial}{\partial x}\left(D \frac{\partial n(x)}{\partial x}\right)-\frac{n(x)^{\beta}-n_{0}^{\beta}}{\tau}+g(x)
$$

where $n$ is the conduction band electron density in the $\mathrm{TiO}_{2}, n_{0}$ is the density in the dark under equilibrium, $D$ and $\tau$ are respectively their diffusion coefficient and lifetime, and $g$ is the local generation rate.

To simplify calculations we assume in the rest of the paper that the electron recombination is linear with electron density $(\beta=1)$. Furthermore, it is assumed that $D$ and $\tau$ and constants independent of the electron density and position in the photoelectrode film.

It should be noted that in typical DSCs, where $\beta<1$, an electron diffusion length $L$ cannot be defined as a constant fundamental parameter, but needs to be discussed in terms of the so-called small perturbation diffusion length $\lambda_{\mathrm{n}}$ that depends on $D, \tau$, and $\beta$, as well as the conduction band electron density that generally varies with position in the film, making $\lambda_{\mathrm{n}}$ position dependent. ${ }^{30}$ However, it has been shown by Barnes and $\mathrm{O}^{\prime}$ Regan that the linear model obtained with $\beta=1$ gives very similar results for the spatial profile of the conduction band electron density, $\eta_{\mathrm{COL}}$ and $\eta_{\mathrm{IPCE}}$, as the non-linear model for a broad range of $\beta$ values, even at the short circuit condition where $\lambda_{\mathrm{n}}$ is strongly position dependent, when the value of the diffusion length $L$ in the linear model is close to the value of $\lambda_{\mathrm{n}}$ calculated for the average electron density. ${ }^{31}$ Moreover, very similar estimates for the electron diffusion length (i.e. $L \approx \lambda_{\mathrm{n}}$ ) are obtained when experimental differences in the $\eta_{\mathrm{IPCE}}$ (socalled IPCE ratio) caused by different illumination geometries are fitted with the linear and non- 
linear models. This suggests that $L$, applied with the linear model, is a good parameter for analyzing the influence of different optical designs on the electron collection efficiency and photocurrent output of DSCs. Nevertheless, although non-ideality represented by the $\beta$-factor can be dropped in a good approximate analysis of short circuit current density, it is needed to reproduce the experimentally observed open circuit voltages. This can be done alternatively by introducing an ideality factor $m$ to the linear model (equation 12 below). To which extent the non-ideal currentvoltage characteristics of DSCs can be ascribed to the $\beta$-factor in one hand and to the $m$-factor on the other hand is presently unclear. For the most general description, both of them can be included in the same model. ${ }^{32}$.[ref Z ] In the present work, we introduce the non-ideality through the $m$-factor only as done before, ${ }^{28}$ and focus our discussion on the electron collection efficiency and short circuit current, for which the linear model is a good approximation, as mentioned above.

With the above assumptions, equation (5) becomes a linear partial differential equation, the solutions of which follow the principle of superposition. Hence, the steady state solution for $\eta_{\mathrm{COL}}$ in the case of any generation profile $g(x)$ can be written as ${ }^{28}$

$$
\eta_{\mathrm{col}}(\lambda)=\frac{\int_{0}^{d} g(x, \lambda) \eta_{\mathrm{col}, \delta}(x) d x}{\int_{0}^{d} g(x, \lambda) d x}
$$

where $d$ is the photoelectrode film thickness. Equation (6) is essentially a weighted average of the collection efficiencies of an electron generated in the film at position $x$, also called as the spatial electron collection efficiency $\eta_{\mathrm{col}, \delta}^{28}$

$$
\eta_{\mathrm{col}, \delta}(x)=\frac{\cosh \left(\frac{d-x}{L}\right)}{\cosh \left(\frac{d}{L}\right)}
$$

where

$$
L=\sqrt{D \tau}
$$

is the electron diffusion length. 
The above model gives us a straightforward way to estimate the combined effects on the light harvesting and electron collection on the photocurrent generation in different light scattering designs: Optical modeling as described in the next section is used to derive the electron generation profile as a function of wavelength, $g(x, \lambda)$, and the overall light harvesting efficiency of the cell, $\eta_{\mathrm{LH}}(\lambda)$, which can then be inserted in equations (6) and (4) to estimate $i_{\text {SC }}$ by equation (3).

Since all the factors in equation (4) and hence the IPCE are wavelength dependent functions, let us define the average collection efficiency integrated over the photoactive region of the dye, $\lambda_{\min } \ldots$ $\lambda_{\max }$, where $\lambda_{\min }=390 \mathrm{~nm}$ and $\lambda_{\max }=830 \mathrm{~nm}$, to help us reformulate equations related to photocurrent generation. The lower end of the range corresponds roughly to the onset of direct badgap excitation of $\mathrm{TiO}_{2}$, which must be avoided to avoid dye degradation in real DSCs by the application of a UV cut-off filter.

The average electron collection efficiency is

$$
\eta_{\mathrm{col}, \mathrm{avg}}=\frac{\int_{\lambda_{\min }}^{\lambda_{\max }} \eta_{\mathrm{col}}(\lambda) \eta_{\mathrm{LH}}(\lambda) \eta_{\mathrm{inj}}(\lambda) \eta_{\mathrm{reg}}(\lambda) \Phi(\lambda) d \lambda}{\int_{\lambda_{\min }}^{\lambda_{\max }} \eta_{\mathrm{LH}}(\lambda) \eta_{\mathrm{inj}}(\lambda) \eta_{\mathrm{reg}}(\lambda) \Phi(\lambda) d \lambda}
$$

And thus, using equation (3) and (4), we can express the short circuit current density simply as

$$
i_{S C}=\eta_{\text {col,avg }} i_{\text {Gen }}
$$

where

$$
i_{\mathrm{gen}}=q_{e} \int_{\lambda_{\min }}^{\lambda_{\max }} \eta_{\mathrm{LH}}(\lambda) \eta_{\mathrm{inj}}(\lambda) \eta_{\mathrm{reg}}(\lambda) \Phi(\lambda) d \lambda
$$

is the electron generation current density per geometric area of the photoelectrode film. The average collection efficiency thus tells us how many percent of the all electrons generated into the $\mathrm{TiO}_{2}$ film by the dye are collected at the short circuit condition

Light scattering has an effect not only on the $i_{\mathrm{SC}}$, but also on the open circuit voltage $\left(V_{\mathrm{OC}}\right)$, fill factor $(F F)$ of the current voltage (IV) curve, and the maximum power output of the solar cell. These 
effects are accounted for by the simplified physical DSC IV model formulated originally by Södergren et al. ${ }^{29}$ based on the continuity equation (5), which can be expressed, using equation (9) as

$$
i_{\text {cell }}\left(V_{\mathrm{PE}}\right)=i_{\text {gen }} \eta_{\text {col,avg }}-i_{\text {rec }, 0} \eta_{\text {col,uni }}\left(e^{-\frac{q_{\mathrm{e}} V_{\mathrm{PE}}}{m k_{\mathrm{B}} T}}-1\right)
$$

where

$$
\eta_{\text {col,uni }}=\frac{\tanh (d / L)}{d / L}
$$

is the collection efficiency of electrons if they were generated uniformly in the film, $k_{\mathrm{B}}$ is the Boltzman constant, $T$ is the absolute temperature, $m$ is the ideality factor, ${ }^{28,29}$ and $i_{\mathrm{REC}, 0}$ is the "dark" recombination current density at the equilibrium, given by

$$
i_{\text {rec }, 0}=\frac{q_{\mathrm{e}} d n_{0}}{\tau}
$$

The expression for the open circuit voltage can be found from equation (12) when $i_{\text {cell }}=0$

$$
V_{\mathrm{OC}}=-\frac{m k_{\mathrm{B}} T}{q_{\mathrm{e}}} \ln \left(\frac{i_{\mathrm{gen}} \eta_{\mathrm{col}, \mathrm{avg}}}{i_{\mathrm{rec}, 0} \eta_{\mathrm{col}, \mathrm{uni}}}+1\right)
$$

We can appreciate from these equations that the $V_{\mathrm{OC}}$ depends not only on the ratio of the generation and and dark recombination current densities, but also on the average collection efficiency that is sensitive to the electron generation profile in the film, and hence, reflects differences in it between the different light scattering designs. To derive the IV curve of the complete DSC, the voltage losses in the other cell components should be taken into account, ${ }^{28}$ but here, we omit these voltage losses and investigate the photoelectrode performance only. The power output $\left(\mathrm{Wm}^{-2}\right)$ of the photoelectrode is simply $P_{\mathrm{TiO} 2}=V_{\mathrm{TiO} 2} i_{\mathrm{CELL}}$, the maximum of which devided by the standard total solar irradiance of $1000 \mathrm{Wm}^{-2}$ defines the energy conversion efficiency $(\eta)$ of the solar cell. The parameter values used in the electrical model calculations were: temperature, $T=293.15 \mathrm{~K}$, exchange current density of the recombination reaction, $i_{\mathrm{REC}, 0}=4.20 \cdot 10^{-4} \mathrm{~A} / \mathrm{m}^{2}$ and the ideality factor $m=2.1{ }^{28}$ The photon flux spectrum, $\phi(\lambda)$, used in calculations is the photon flux of the AM1.5G $\left(1000 \mathrm{~W} / \mathrm{m}^{2}\right.$ solar spectral irradiance) spectrum. The electron diffusion length $(L)$ was varied from $1 \mu \mathrm{m}$ to $14 \mu \mathrm{m}$. Note that the variation of $L$ corresponds to variation of the electron diffusion coefficient $D$ since the electron 
lifetime $\tau$ was kept constant by fixing the value of $i_{\text {rec }, 0}$ constant. This represent the situation where the electron diffusion lentgh is limited by poor electron transport properties of the photoelectrode film due for example to a low temperature method used in the preparation of the film on a flexible plastic substrate.

\section{Results and discussion}

Figure 3 displays the light harvesting efficiencies $\eta_{\mathrm{LH}}(\lambda)$ calculated for a type I cell used as reference (black lines in both Figures 3(a) and 3(b)), and the optimized designs found for type II (red dashed line) and type III (blue dotted line) cells. Compared to the standard cells, $\eta_{\mathrm{LH}}(\lambda)$ of all modified photoelectrode films is improved. It can be readily seen that the highest optical absorptance is attained for type III cell. The main reason for this is the competing strong diffuse reflectance introduced by the scatterers mixed with paste in type II cell, which balance the positive effect of light trapping. This is particularly noticeable in spectral regions at which absorptance of the standard reference type I cell is already high, i.e., green and blue ranges, for which either no effect or even a slight reduction of $\eta_{\mathrm{LH}}(\lambda)$ is expected, as it can be seen in Figure 3(a). This is not the case for type III cells, where the diffuse scattering layer always increases $\eta_{\mathrm{LH}}(\lambda)$ irrespective of the photon wavelength. In fact, these results are in excellent qualitative and quantitative agreement with reported experimental ones. ${ }^{33},{ }^{34}$ However, as we will show below, this better performance of type III cell versus type II as light harvester does not imply that better conversion efficiency will be attained in all cases. 


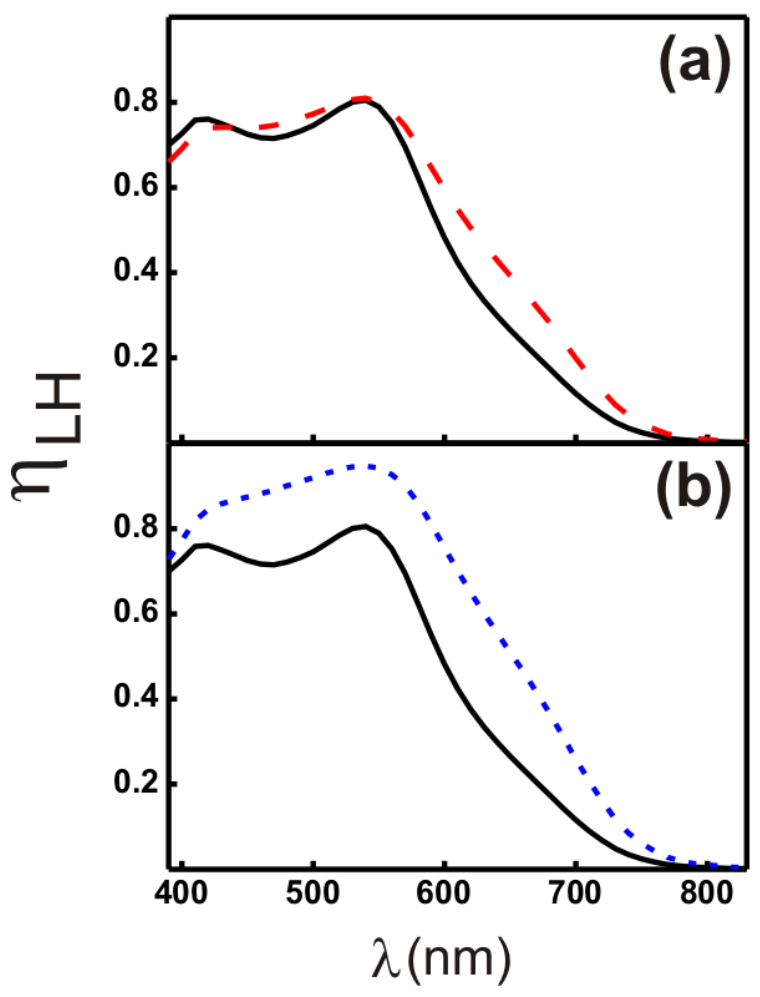

Figure 3. Spectral light harvesting efficiency $\left(\eta_{\mathrm{LH}}\right)$ or optical absorptance of (a) a type II cell (red dashed line) and (b) a type III cell (dotted blue line). The absorptance spectra of a standard semitransparent reference type I cell is also plotted for the sake of comparison (black solid lines).

In Figure 4 we plot the spatial light absorption profile or electron generation function $g(x, \lambda)$ for the three types of cell under consideration. Different wavelengths were chosen to illustrate the different effect the optical designs may have on this parameter. The first conclusion that can be reached when analyzing these curves is that the profiles strongly deviates from the classical expression derived from Lambert-Beer law $g(x, \lambda)=\alpha_{A} \cdot \exp \left(-\alpha_{A} \cdot x\right)$. Although clear differences can be observed for photons of all energies, it is indeed for those wavelengths that are more weakly absorbed by the dye (see Figure 4(c) for $\lambda=650 \mathrm{~nm}$ ) that maximum harvesting is achieved either somewhere inside the cell, as it happens for type II cell, (red dashed line) or even at the end of the absorbing electrode, as it occurs for type III cell, (blue dotted line). Interestingly, type II cells, which are comparatively less efficient as overall light harvesters than type III ones, favors the concentration of photogenerated electrons close to the collecting contact. Contrarily, type III cells, for which absorption is greatly enhanced for photons of all wavelengths, tend to shift the maximum of the electron generation function farther away from the collection point. 


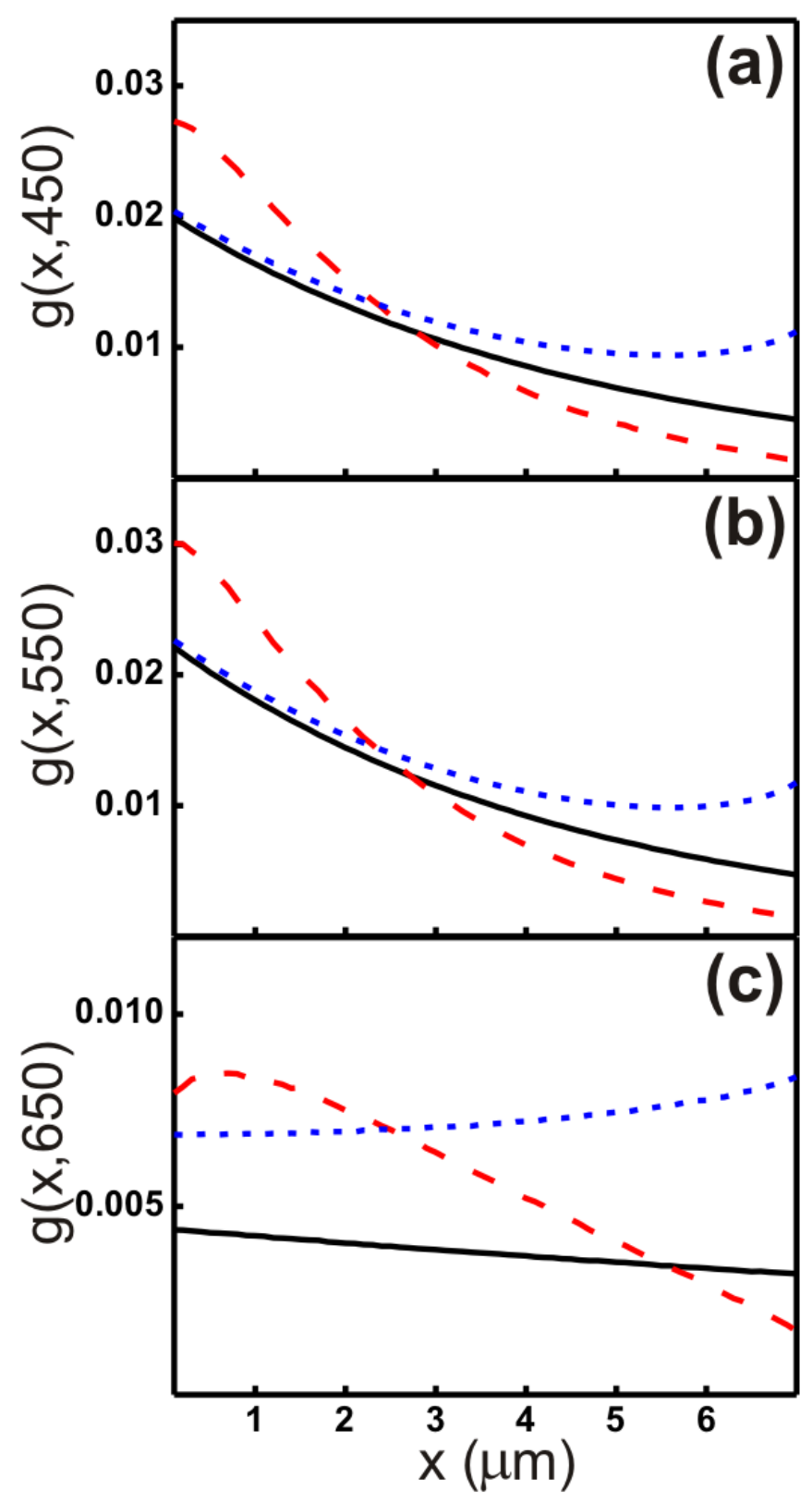

Figure 4. Electron generation function $(g(x, \lambda))$ or spatial optical absorptance profile for type I (black solid lines), type II (red dashed lines) and type III (dotted blue line) cells calculated for three different wavelengths, namely, (a) $\lambda=450 \mathrm{~nm}$, (b) $\lambda=550 \mathrm{~nm}$, and (c) $\lambda=650 \mathrm{~nm}$.

The electron collection efficiency $\eta_{\mathrm{COL}}(\lambda)$ depends on the spatial dependence of $g(x, \lambda)$ and the electron diffusion length $L$ as indicated in equations (6) and (7), which we use to draw the different curves shown in Figure 5. These graphs demonstrate that the optical design that optimizes electron collection is that of type II cells independently of the photon wavelength considered. As expected, for a fixed electrode thickness $d$, as $L$ increases, the magnitude of the effect of the electron 
generation profile $g(x, \lambda)$ on $\eta_{\mathrm{COL}}$ diminishes, being almost null when $L>d$. By comparing Figures 3 and 5 , it can be concluded that, for $L<d$, it is not possible to optimize $\eta_{\mathrm{LH}}(\lambda)$ and $\eta_{\mathrm{COL}}(\lambda)$ simultaneously within just one of the optical designs herein analyzed. As we will show next, it implies that different optical designs will be the optimal ones depending on the relation between electron diffusion length and electrode thickness.

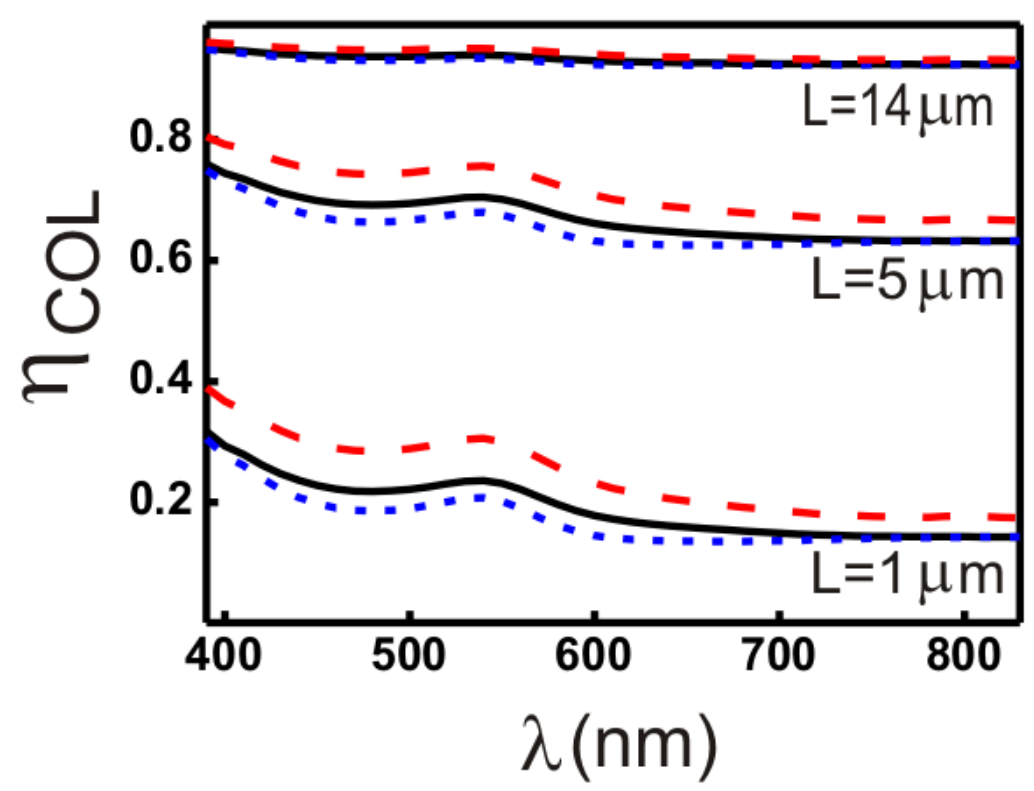

Figure 5. Collection efficiency as function of wavelength for different electron diffusion lengths $L$. Top three curves are for $L=14 \mu \mathrm{m}$, middle three curves for $L=5 \mu \mathrm{m}$ and botton three curves for $L=1$ $\mu \mathrm{m}$. Results are shown for type I (black solid lines), type II (red dashed lines) and type III (dotted blue line) cells.

In Figures 6 and 7 it is shown, respectively, the calculated incident photon to collected electron

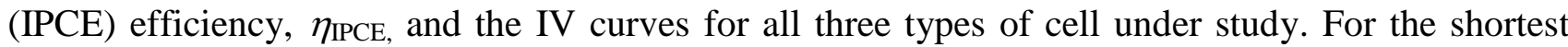
electron diffusion length considered, $L=1 \mu \mathrm{m}<<d$, maximum $\eta_{\mathrm{IPCE}}$ and hence short circuit photocurrent (current at $\mathrm{V}=0$ ), $i_{S C}$, are attained for type II cell, since $\eta_{\mathrm{LH}, \mathrm{II}}<\eta_{\mathrm{LH}, \mathrm{III}}$ while $\eta_{\mathrm{COL}, \mathrm{II}}>\eta_{\mathrm{COL}, \mathrm{III}}$. Contrarily, when $L=14 \mu \mathrm{m}>d$, type III cells present the best performance, since $\eta_{\mathrm{LH}, \mathrm{III}}>\eta_{\mathrm{LH}, \mathrm{II}}$ and $\eta_{\mathrm{COL}, \mathrm{III}} \approx \eta_{\mathrm{COL}, \mathrm{II}}$. Interestingly, when $L$ takes values that are below but on the order of the electrode thickness, the need for a compromise between efficient collection and light harvesting becomes more evident. Thus, for $L=5 \mu \mathrm{m}$, the increase of absorptance in type III cells, $\eta_{\mathrm{LH}, \mathrm{III}}>\eta_{\mathrm{LH}, \mathrm{II}}$, compensates its worse electron collection, $\eta_{\mathrm{COL}, \mathrm{II}}>\eta_{\mathrm{COL}, \mathrm{III}}$ and gives rise to higher $\eta_{\mathrm{IPCE}}$ and $i_{S C}$ than those that would obtained in type II cells. 


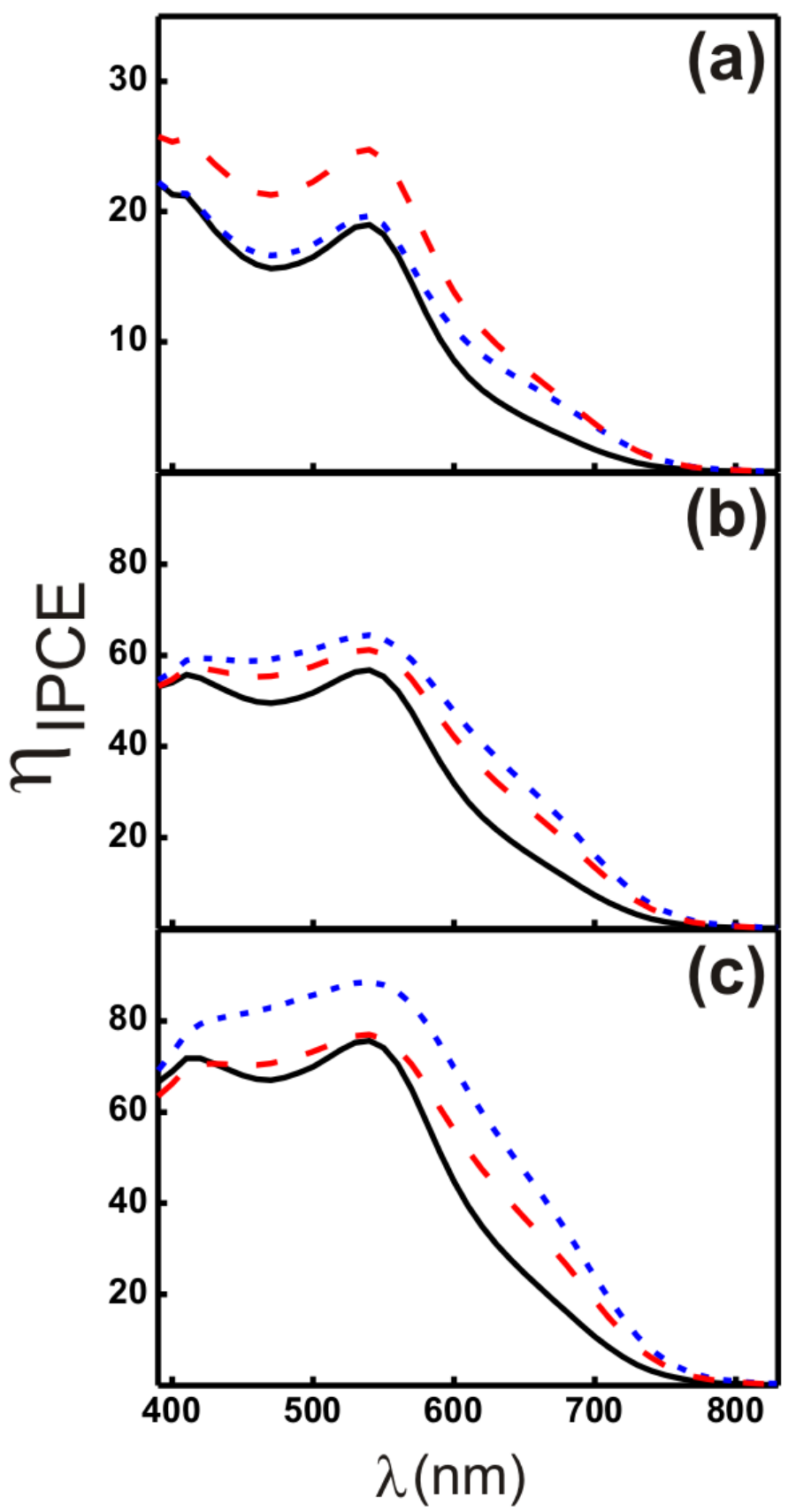

Figure 6. IPCE for three different electron diffusion lengths, namely, (a) $L=1 \mu \mathrm{m}$, (b) $L=5 \mu \mathrm{m}$, and (c) $L=14 \mu \mathrm{m}$. Results are shown for type I (black solid lines), type II (red dashed lines) and type III (dotted blue line) cells. 


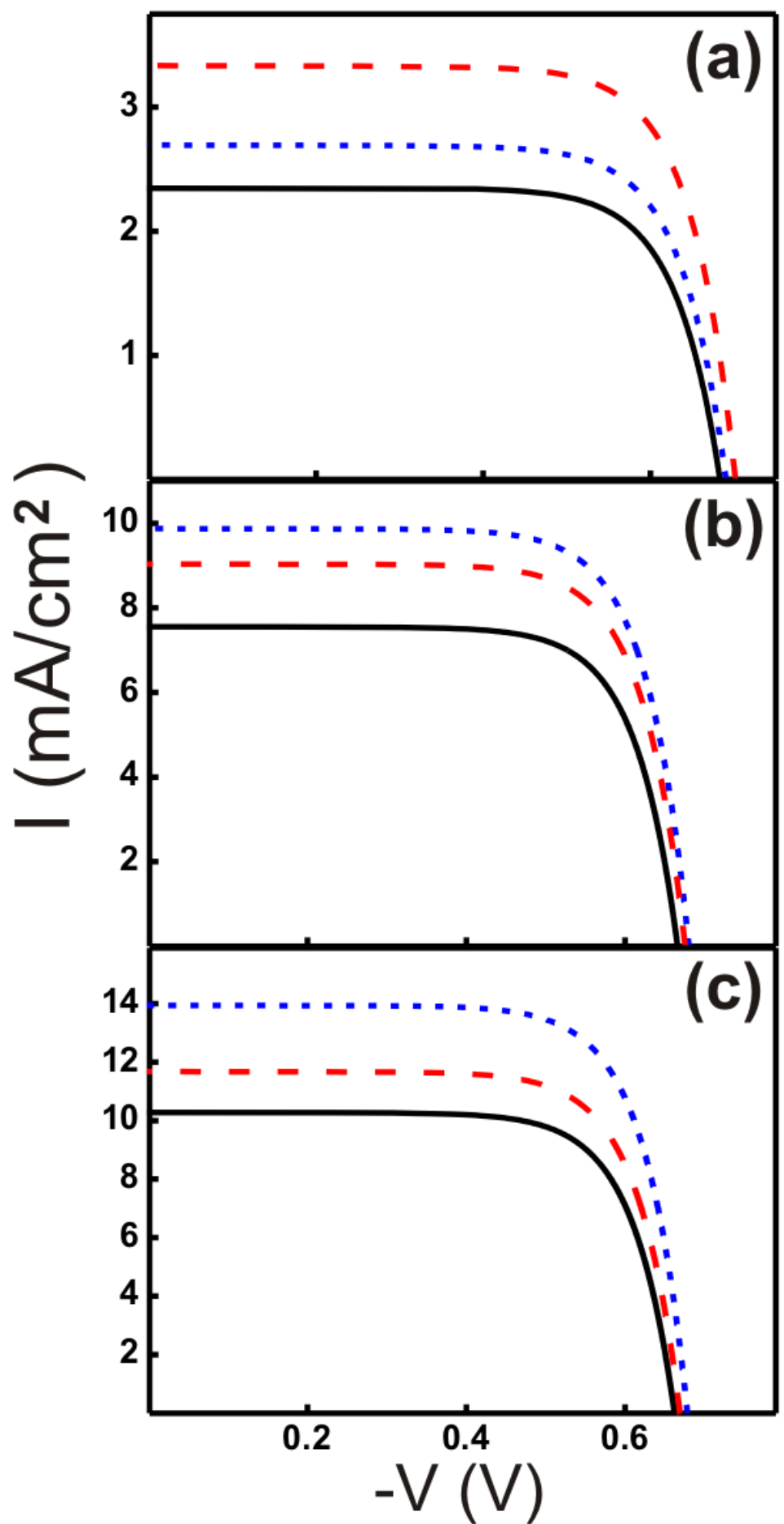

Figure 7. Current density-voltage (IV) curves for three different electron diffusion lengths, namely, (a) $L=1 \mu \mathrm{m}$, (b) $L=5 \mu \mathrm{m}$, and (c) $L=14 \mu \mathrm{m}$. Results are shown for type I (black solid lines), type II (red dashed lines) and type III (dotted blue line) cells. 
Although much less significant than the enhancement of the photocurrent, diffuse scattering layers or embedded particles also cause a slight increase of the open circuit photovoltage, as it can be seen in Figure 7. In DSCs, $V_{\mathrm{OC}}$ results from the accumulation of conduction band electrons as a result of the balance between electron generation and recombination per unit volume of the $\mathrm{TiO}_{2}$ film. However, it is also affected by the generation profile through its effect on the average collection efficiency, as expressed in equation (15). So, improving the overall light harvesting efficiency $\eta_{\mathrm{LH}, \mathrm{avg}}$ of the photoelectrode film by means of the optical design, implies an increase of $V_{\mathrm{OC}}$, since it rises the mean electron density in the film due to the larger generation rate by unit volume. The effect of electron diffusion length $L$ on $V_{\mathrm{OC}}$ is less straightforward but as equation (12) shows, it arises from the non-uniform electron generation profile. When the electron generation is biased towards the collecting contact, there will be a constant flux of electrons diffusing from the region near to the contact deeper into the film. This corresponds to a gradient in the electron density that is the steeper the smaller the electron diffusion coefficient $D$. In the films with mixed scattering particles, electron generation is biased relatively more towards the contact than in the stardard case, while the opposite is true for the coupled scattering layer. This explains tendency of the scattering particles to produce higher $V_{\mathrm{OC}}$ compared to the other cases at same $L$ (remember that here $L$ was varied by varying $D$, while keeping $\tau$ constant).

Although we have centred our discussion on a standard $7 \mu \mathrm{m}$ thickness electrodes, similar conclusions can be extracted for thinner nanocrystalline layers. Values of $i_{S C}, V_{O C}, F F$ and total conversion efficiency are listed for optimized optical designs for different electrode thickness $d$ in table 1 . In all cases, the introduction of scattering particles or the coupling of a scattering layer gives rise to an increased efficiency, although the enhancement with respect to type I cells is more dramatic for thin electrodes since less light is absorbed by the reference. The interplay between $L$ and $d$ also yields some interesting trends for $L=1 \mu \mathrm{m}$. In this particular case, both type I and type II cells show an increase of efficiency as the film thickness increases, while type III cell displays the opposite trend. This is a consequence of the very different way in which electron generation takes place in each configuration. In both type I and II $g(x, \lambda)$ shows its maximum at $x \approx 0$, i.e., close to the contact, while in type III $g(x, \lambda)$ is bended upwards far from the collection point. Such effect does not affect $\eta_{C O L}$ significantly in very thin cells, so their best performance is also found when the type III design is considered, as it happens for thicker electrodes when higher values of $L$ are considered. As the thickness is increased, the efficiency of type III design drops dramatically as $\eta_{C O L, I I I}$ does. For 
thicker ones, $\eta_{C O L, I I}>\eta_{C O L, I>}>\eta_{C O L, I I I}$ and type II configuration becomes the optimum one, as it was shown above.

\begin{tabular}{|c|c|c|c|c|c|c|c|c|c|c|c|c|c|}
\hline \multirow[t]{2}{*}{$d / \mu \mathrm{m}$} & \multirow[t]{2}{*}{$L / \mu \mathrm{m}$} & \multicolumn{3}{|c|}{$i_{S C} / \mathrm{mA} \mathrm{cm}^{-2}$} & \multicolumn{3}{|c|}{$V_{O C} / \mathrm{V}$} & \multicolumn{3}{|c|}{ FF $1 \%$} & \multicolumn{3}{|c|}{$\eta 1 \%$} \\
\hline & & Type I & Type II & Type III & Type I & Type II & Type III & Type I & Type II & Type III & Type I & Type II & Type III \\
\hline 2 & 1 & 2.34 & 3.03 & 4.09 & 0.62 & 0.63 & 0.65 & 72 & 73 & 73 & 1.05 & 1.39 & 1.94 \\
\hline 4 & 1 & 2.36 & 3.25 & 3.18 & 0.65 & 0.67 & 0.67 & 73 & 74 & 74 & 1.13 & 1.60 & 1.57 \\
\hline 7 & 1 & 2.35 & 3.33 & 2.69 & 0.68 & 0.70 & 0.69 & 74 & 74 & 74 & 1.19 & 1.74 & 1.38 \\
\hline 2 & 5 & 4.42 & 5.52 & 8.17 & 0.62 & 0.63 & 0.65 & 72 & 73 & 73 & 1.97 & 2.51 & 3.87 \\
\hline 4 & 5 & 6.63 & 8.18 & 10.22 & 0.64 & 0.66 & 0.67 & 73 & 73 & 74 & 3.12 & 3.93 & 5.02 \\
\hline 7 & 5 & 7.55 & 9.03 & 9.87 & 0.67 & 0.68 & 0.68 & 74 & 74 & 74 & 3.70 & 4.51 & 4.96 \\
\hline 2 & 14 & 4.62 & 5.74 & 8.54 & 0.62 & 0.63 & 0.65 & 72 & 72 & 73 & 2.05 & 2.61 & 4.05 \\
\hline 4 & 14 & 7.65 & 9.31 & 11.96 & 0.64 & 0.65 & 0.67 & 73 & 73 & 74 & 3.59 & 4.46 & 5.88 \\
\hline 7 & 14 & 10.27 & 11.67 & 13.94 & 0.66 & 0.67 & 0.68 & 73 & 74 & 74 & 5.00 & 5.75 & 6.99 \\
\hline
\end{tabular}

Table 1. Calculated short circuit photocurrents $\left(i_{S C}\right)$, open circuit photovoltages $\left(V_{O C}\right)$, fill factor $(F F)$ and efficiencies $(\eta)$ for the different types of DSCs considered varying electrode thickness $(d)$ and electron diffusion length $(L)$.

In practical terms, DSC commonly built both at research and commercial levels are prepared by sintering at high temperatures $\left(\mathrm{T}>400{ }^{\circ} \mathrm{C}\right)$ the $\mathrm{TiO}_{2}$ nanocrystals before sensitizing them with dyes, which results in good mass continuity and enhanced electron transport throughout the porous semiconductor network. For these cells, electrodes are typically on the order of several microns and electron diffusion lengths of $L>20 \mu \mathrm{m}$ are claimed. ${ }^{35}$ On the other hand, in order to build DSCs on plastic substrates aiming at developing flexible and/or transparent photovoltaic devices, low sintering temperatures are required, yielding a much shorter electron diffusion length. ${ }^{36}$ Results presented in Figures 6 and 7 explain why no record DSC has been made by using scattering particles embedded within the absorbing electrode, but only by coupling a diffuse scattering layer to the $\mathrm{nc}^{-\mathrm{TiO}_{2}}$ electrode (Figure 7(c)). Also, they provide a guide to achieve maximum efficiency in cells in which 
the electron diffusion length is limited by the preparation conditions, being possible to state that for very short $L<<d$ the design herein referred to as type II should always be the preferred one, while for intermediate values $L \approx d$ the best choice must be decided by careful inspection of the structure.

It should be remarked that the effect of the light scattering design in type I and type II cells had been compared before, an similar conclusions to the ones herein reported had been reached. However, the performance of a coupled back scattering layer (type III cell) was not accounted for, nor a rigorous comparison established between the different designs. Most importantly, the effect of the interplay between the optical absorption profile and the electron collection efficiency had been only barely considered, while in our case we provide results that help to establish what sort of optical design should prevail depending on this interplay.

\section{Conclusions}

In this paper, we have introduced a combined optical and electrical modeling to investigate how different commonly used diffuse light scattering designs influence the conversion efficiency of dye sensitized solar cells. We aimed at filling a hole that apparently existed in the field of modeling of this kind of photovoltaic devices, since although the effect on light harvesting efficiency of scattering layers seemed to be widely recognized and indirectly accounted for in calculations, the strong modification of the electron generation function and thus of the electron collection efficiency seemed to have been overlooked. The reason for this is likely the long electron diffusion lengths (longer than the electrode thickness) that are typically obtained in the optimized cells. Our analysis explain why record cells can only be made by coupling diffuse scattering layers acting as back reflectors rather than mixing scattering particles within the absorbing paste. Interestingly, however, for electron diffusion length shorter than the electrode thickness, embedding diffuse scattering particles in the nanocrystalline paste may yield a better output even when the light harvesting is not optimal, since the electron generation function largely increases at distances close to the metal contact with respect to any other configuration, which boost the collection efficiency compensating for the smaller absorptance. This finding becomes particularly relevant when low sintering temperature pastes, which usually imply short electron diffusion lengths, are used, as it is the case of flexible dye solar cells deposited on conducting plastic substrates. We believe our results will serve not only to explain previous observations, but also to identify guidelines to optimize the use of light in different sorts of dye solar cells.

\section{Acknowledgements}


HM thanks the Spanish Ministry of Science and Innovation for funding provided under grants MAT2008-02166, MAT2011-23593, and CONSOLIDER HOPE CSD2007-00007, to Junta de Andalucía for grants FQM3579 and FQM5247. HM and JH thank funding under a grant from Nordic Innovation Centre (NICe). 


\section{References}

1 M. Grätzel, Nature 2001, 414, 338.

2 N.G. Park, Korean J. Chem. Eng. 2010, 27, 375.

3 S. Ito, S.M. Zakeeruddin, R. Humphry-Baker et al., Adv. Mater. 2006, 18, 1202.

4A.G. Agrios, I. Cesar, P. Comte, M.K. Nazeeruddin, M. Grätzel, Chem. Mater. 2006, 18, 5395.

5 K.M. Lee, V. Suryanarayanan, K.C. Ho, Sol. Energy Mater. Sol. Cells 2006, 90, 2398.

6 Z.S. Wang, H. Kawauchi, T. Kashima, H. Arakawa, Coor. Chem. Rev. 2004, 2481381.

7 F. Huang, D. Chen, X.L. Zhang, R.A. Caruso, Y.B. Cheng, Adv. Funct. Mater. 2010, 20, 1301.

8 F. Gao, Y. Wang, D. Shi, J. Zhang, M. Wang, X. Jing, R. Humphry-Baker, P. Wang, S.M.

Zakeeruddin, M. Grätzel, J. Am. Chem. Soc. 2008, 130, 10720.

9 Y. Chiba, A. Islam, R. Komiya, N. Koide, L. Han, App. Phys. Lett. 2006, 88, 223505.

10 D. Shi, N. Pootrakulchote, R. Li, et al. J. Phys. Chem. C 2008, 112, 17046.

11 C.J. Barbé, F. Arendse, P. Comte, M. Jirousek, F. Lenzmann, V. Shklover, M. Grätzel, J. Am. Ceram. Soc. 1997, 80, 3157.

12 J. Xi, Q. Zhang, K. Park, Y. Sun, G. Cao, Electrochimica Acta 2011, 56, 1960.

13 Z. Tian, H. Tian, X. Wang et al., App. Phys. Lett. 2009, 94, 031905.

14 S. Hore, P. Nitz, C. Vetter, C. Prahl, M. Niggemann, R. Kern, Chem. Commun. Cambridge 2005, 2011.

15 H.J. Koo, Y.J. Kim, Y.H. Lee, W.I. Lee, K.Kim, N.G. Park, Adv. Mater. Weinheim, Ger. 2008, 20, 195.

16 J. Bisquert, I. Morá-Seró, J. Phys. Chem. Lett. 2010, 1, 450.

17 J. Villanueva, J.A. Anta, E. Guillén, G. Oskam, J. Phys. Chem. C 2009, 113, 19722.

18 S. Wenger, M. Schmid, G. Rothenberger, A. Gentsch, M. Grätzel, J.O. Schumacher, J. Phys. Chem. C 2011, 115, 10218.

19 J. Halme, G. Boschloo, A. Hagfeldt, P. Lund, J. Phys. Chem. C 2008, 112, 5623.

20 P. R. F Barnes, A.Y. Anderson, S.E.Koops, J. Durrant, B. O’Regan, J. Phys. Chem. C 2009, 113, 1126.

21 L.M. Peter, J. Phys. Chem. C 2007, 111, 6601.

22 J.P. González-Vázquez, J.A. Anta, J. Bisquert, J. Phys. Chem. C 2010, 114, 8552.

23 A. Usami, Chem. Phys. Lett. 1997, 277, 105.

24 J. Ferber, J. Luther, Sol. Energy Mater. Sol. Cells 1998, 54, 265.

25 G. Rothenberger, P. Comte, M. Grätzel, Sol. Energy Mater. Sol. Cells 1999, 58, 321.

26 A. Usami, Sol. Energy Mater. Sol. Cells 2000, 64, 73. 
27 Z. Zhang, S. Ito, B. O’Regan, et al., Z. Phys. Chem. 2007, 221, 319.

28 J. Halme, P. Vahermaa, K. Miettunen, P. Lund, Adv. Mater. 2010, 22, E210.

29 S. Södergren, A. Hagfeldt, J. Olsson, S. Lindquist, J. Phys. Chem. 1994, 98, 5552.

30 Bisquert, J., Mora-Seró, I., J.Phys.Chem.Lett. 2010, 1, 450-456

31 Barnes, P. R. F., O’Regan, B. C., J.Phys.Chem. C, 2010, 19134-19140

32 Barnes, P. R. F., Anderson, A. Y., Durrant, J. R., O’Regan, B., Phys.Chem.Chem.Phys., 2011, 13, 5798-5816.

33 I.G. Yu, Y.J. Kim, H.J. Kim, C. Lee, W.I. Lee, J. Mater. Chem. 2011, 21, 532.

34 H.J. Koo, J. Park, B. Yoo, K. Yoo, K. Kim, N.G. Park, Inorganica Chimica Acta 2008, 361, 677.

35 A.C. Fisher, L.M. Peter, E.A. Ponomarev, A.B. Walker, K.G.U. Wijayantha, J. Phys. Chem. B 2000, 104, 949.

36 M. Toivola, J. Halme, K. Miettunen, K. Aitola, P. Lund, Int. J. Ener. Res. 2009, 33, 1145. 